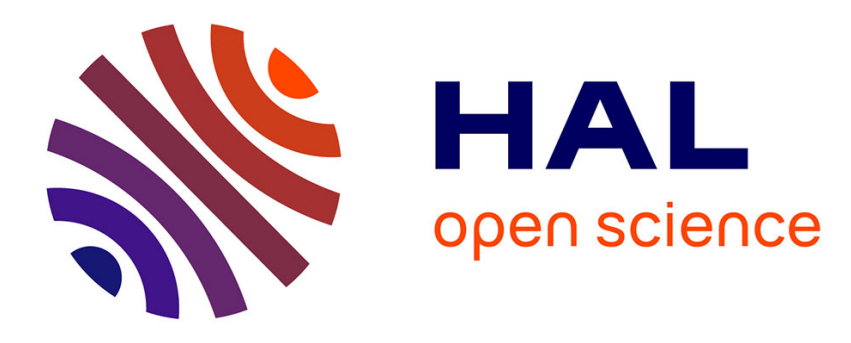

\title{
Positions of the secular resonances in the primordial Kuiper Belt disk
}

\author{
Daniel Baguet, Alessandro Morbidelli, Jean-Marc C. Petit
}

\section{To cite this version:}

Daniel Baguet, Alessandro Morbidelli, Jean-Marc C. Petit. Positions of the secular resonances in the primordial Kuiper Belt disk. Icarus, 2019, 334, pp.99-109. 10.1016/j.icarus.2019.113417 . hal02736885

\section{HAL Id: hal-02736885 \\ https://hal.science/hal-02736885}

Submitted on 24 Nov 2020

HAL is a multi-disciplinary open access archive for the deposit and dissemination of scientific research documents, whether they are published or not. The documents may come from teaching and research institutions in France or abroad, or from public or private research centers.
L'archive ouverte pluridisciplinaire HAL, est destinée au dépôt et à la diffusion de documents scientifiques de niveau recherche, publiés ou non, émanant des établissements d'enseignement et de recherche français ou étrangers, des laboratoires publics ou privés. 


\title{
Positions of the secular resonances in the primordial Kuiper Belt disk
}

\author{
D. Baguet ${ }^{\mathrm{a}}$, A. Morbidelli ${ }^{\mathrm{b}}$, J.-M. Petit ${ }^{\mathrm{a}}$ \\ anstitut UTINAM, UMR 6213, CNRS, Université Bourgogne Franche-Comté, OSU Theta \\ F-25000, Besançon, France \\ ${ }^{b}$ Laboratoire Lagrange, UMR 7293, Université Côte d'Azur, CNRS, Observatoire de la Côte \\ d'Azur, Boulevard de l'Observatoire, 06304 Nice Cedex 4, France
}

\begin{abstract}
1 The dynamically hot and cold populations of the Kuiper Belt probably formed 2 from two distinct regions of the Solar System. The former originated from a 3 massive planetesimal disk extending from the primordial position of Neptune to $4 \sim 30$ AU and the latter from a light extension of the planetesimal disk, prolong5 ing beyond 30 AU. Previous studies on the dynamical evolution of the primordial 6 cold population only accounted for the giant planets and did not consider its 7 evolution under the influence of the massive part of the planetesimal disk. The 8 latter affects it only indirectly through its interactions with the giant planets.

9 Our goal is to introduce the perturbation of the massive part of the planetes10 imal disk on the apsidal and nodal precessions of both the giant planets and 11 planetesimals, using the linear secular theory. We want to see how it affects the 12 positions of the secular resonances. In the first place, we look at the positions 13 of the secular resonances after the disappearance of the solar nebula, when the 14 giant planets were locked in a multiresonant configuration. Because of this mul15 tiresonant configuration, the linear secular theory allows us to compute only 16 the nodal part. The existence of a massive disk of planetesimals makes the $f_{5}$ 17 frequency non-zero. We show that the associated secular resonance is located in 18 the region corresponding to the current cold Kuiper Belt in several multireso19 nant configurations of the giant planets. The efficiency of this secular resonance 20 in rising the inclinations of the objects depends on the misalignment between 21 the total angular momentum of the giant planets and the direction orthogonal 22 to the massive planetesimal disk. If both are aligned, the amplitude associated 23 to the $f_{5}$ frequency is null and the resonance has no effect. We illustrate this
\end{abstract}

Email address: daniel.baguet@utinam.cnrs.fr (D. Baguet) 

44 Sun.

with simple numerical integrations, where the nodal precessions exerted by the massive disk is mimicked using fictitious forces. Then, we look at the positions of the apsidal and nodal secular resonances just before the instability between the giant planets. We find that taking into account the massive part of the disk only shifts the secular resonances by less than $1 \%$ compared with the case where we do not account for it and the $f_{5}$ secular resonance is located beyond $150 \mathrm{AU}$. Keywords: celestial mechanics, Kuiper Belt, secular resonance

\section{Introduction}

The Kuiper Belt is the residual disk of a more massive primordial planetesimal disk. The Kuiper Belt objects, in their orbital structure, keep a trace of the past dynamical evolution of the giant planets. Studying their dynamics is therefore important to acquire a better understanding of the dynamical evolution of the giant planets during the life of the Solar System.

It has been shown that before the dissipation of the protoplanetary gas disk, the giant planets should have followed an inward migration until they got locked in a multiresonant configuration where each planet is in mean motion resonance (MMR) with the others (Masset and Snellgrove (2001), Morbidelli et al. (2007)), with a preference for the configuration where Jupiter and Saturn are in a 3:2 MMR because it allows their gaps in the protoplanetary gas disk to overlap. This overlap of the gaps is needed to explain why our solar system does not contain a hot Jupiter, because it refrains Jupiter from migrating close to the

From this multiresonant configuration, the giant planets, by interacting with the planetesimal disk, were driven in a migration phase until they reached their current positions and during which a dynamical instability among them occurred. This instability, which has been proposed in the framework of the Nice model (Tsiganis et al. (2005), Morbidelli et al. (2005), Gomes et al. (2005)) and the planetesimal-driven migration allow to reproduce many of the orbital features of the Kuiper Belt (Nesvorný, 2018).

In order to explain the difference in color, size distribution and binary fraction between the dynamically hot and the dynamically cold populations in the classical Kuiper Belt, it has been suggested that the hot population formed in 
a region between Neptune and $30 \mathrm{AU}$, from a primordial massive planetesimal disk of mass in the range $\sim 10-60 M_{\oplus}$ (Gomes (2003), Levison et al. (2008), Nesvorný and Vokrouhlický (2016)). The outer edge of this disk is placed at 30 AU because it allows to stop at this location the planetesimal-driven migration of Neptune which corresponds to its current semi-major axis (Gomes et al., 2004). In other respects, the cold classical population is probably the remnant of a lighter planetesimal disk formed in-situ (Nesvorný, 2015b). Besides, numerical integrations that tried to reproduce the cold Kuiper Belt from a single massive disk extending from the primordial position of Neptune to 50 AU did not succeed to reach a sufficient rate of mass depletion in the region of the current cold Kuiper Belt (Gomes et al., 2018). However, it is still unclear where the inner and the outer edge of this last one were located and why there is this sharp transition between the massive planetesimal disk and the light planetesimal disk.

In the rest of this paper, we will call the massive disk (MD) the part of the planetesimal disk between Neptune and 30 AU, and the light disk (LD) the part of the planetesimal disk beyond $30 \mathrm{AU}$.

The cold classical Kuiper Belt allows us to put strong constraints on the dynamical evolution of the giant planets and in particular Neptune (Batygin et al. (2011), Dawson and Murray-Clay (2012)), because the latter must migrate in such a way that the hot population forms from the MD while keeping the $\mathrm{LD}$ located in the region corresponding to the current classical Kuiper Belt cold enough by not exciting it. Given that the MD has a mass comparable to that of an ice giant planet, it can have a significant influence on the dynamics of an object in the LD and in particular, it will bring a perturbation on the apsidal and nodal precessions of the orbits of the planets and of the small bodies. This can have an important effect in determining the positions of the secular resonances. The latter occur where the frequency of the free apsidal or nodal precessions of a small body, depending on its semi-major axis, equals one of the eigenfrequencies of the apsidal or nodal precessions of the planetary system, respectively. Batygin et al. (2011) did numerical integrations where they follow the evolution of the MD and of the LD. In their paper, it is said that the presence of the massive Kuiper Belt enhanced the free precession of the Kuiper 
Belt objects, so it suggests that the MD objects act on the LD objects. However, they did their numerical integrations by using the Mercury6 integration software package. Yet, in the standard version of Mercury 6 the planetesimals of the disk do not interact with each other. Therefore, it is likely that in their numerical integrations the MD does not directly perturb the LD objects but that the latter see only the indirect effect through the action of the planets. On the other hand, Dawson and Murray-Clay (2012) analytically explored the excitation of the eccentricities of the LD objects caused by Neptune alone but where the other giant planets indirectly act on the LD objects through their influence on the apsidal precession of Neptune. However, they did not include the contributions of the other giant planets and of the MD to the apsidal precession of the LD objects. In their numerical study, where only Neptune is present, they included fictitious forces on the ice giant so as to reproduce its radial migration and its eccentricity damping under the effect of the interactions with the planetesimal disk as well as its apsidal precession. The MD itself is represented by a set of massless particles and does not act on the LD objects. Nesvorný (2015b) included fictitious forces on the giant planets so as to reproduce their radial migrations and their eccentricity and inclination damping but the MD is not present so it does not directly affect the apsidal and nodal precessions of the giant planets and of the LD objects

Thus, in this work we investigate the positions of the secular resonances by considering both the effects of the MD on the giant planets and on the LD objects. In order to do so, we represent the MD by an axisymmetric continuous thick disk and we take into account its contribution to the apsidal and nodal precession frequencies in the linear secular theory.

Studies of the positions of the secular resonances and of their effects in the Kuiper Belt under the perturbation of the gravitational potential of a disk have already been made by Nagasawa and Ida (2000) and Li et al. (2008). They showed that the locations of the secular resonances due to the giant planets are shifted when they account for the solar nebula. They modeled the latter based on the minimum mass solar nebula of Hayashi (1981). Then, the depletion of the nebula causes the sweeping of the secular resonances in the Kuiper Belt. With the current orbital architecture of the giant planets, they showed that if 
the exponential depletion timescale is $10^{7} \mathrm{yr}$, the sweeping secular resonances are efficient in exciting the eccentricities and inclinations of the Kuiper Belt objects at the observational level, provided that the inclination between the invariant plane of the planets and the midplane of the solar nebula is high enough. Moreover, with a more compact orbital architecture of the giant planets, they showed that the exponential depletion timescale has to be $10^{8}$ yr. However, the lifetime of gas nebulae around stars is of the order of $10^{6}-10^{7} \mathrm{yr}$ (Haisch et al. (2001), Mamajek (2009)). Thus, with a compact orbital configuration, this mechanism cannot be sufficient to rise the eccentricities and inclinations of the Kuiper Belt objects. Besides, the mass distribution of the primordial planetesimal disk is not the same as the mass distribution of the solar nebula and the former is much less massive than the latter, while still as massive as the ice giant planets. Hence, we think that it is worthwhile to explore the positions of the secular resonances caused by the gravitational effects of the primordial planetesimal disk.

We present the method used in the next section. In section 3, we explore the positions of the secular resonances during the period when the giant planets were placed in a multiresonant configuration and the timescale needed to excite an object located in it. As it will be explained in the concerned section, we can only look at the nodal secular resonances in such multiresonant configurations. In section 4, we explore the positions of the secular resonances just before the dynamical instability. We conclude in section 5 .

\section{Method}

We base our approach on the linear secular theory (see for example Murray and Dermott (2000), chapter 7, for details). Given the gravitational potential of a disk, the secular theory allows us to determine its contribution to the apsidal and nodal precessions of bodies. We model the MD as an axisymmetric continuous thick disk with a planar symmetry and centered on the Sun. We arbitrarily choose the reference frame such that its origin is also centered on the Sun and such that the plane $(O x y)$ corresponds to the plane of symmetry of the MD. Consequently, the MD has a radial density profile symmetric around the $z$-axis and a vertical density profile symmetric in regards to the plane $z=0$. In 
the following, the inclinations of the bodies are referred with respect to the latter plane. With the help of the numerical method developed by Fukushima (2016) we calculate the gravitational potential induced by the MD from its density profile. In the linear secular theory, the disturbing function developed to the second order in eccentricities and in inclinations is the gravitational potential averaged on one orbital period along a fixed ellipse. Therefore, in order to include the dynamical effect of the MD in the linear secular theory, we calculate its contribution to the second order disturbing functions of the giant planets and of a LD object, which is considered as a massless particle. For that, in the first place, for each of the giant planets, let us consider its total disturbing function $\mathcal{R}_{j}^{\text {tot }}$ where the subscript $j$ denotes the $j$ th giant planet, from the closest to the Sun to the outermost. We can write $\mathcal{R}_{j}^{\text {tot }}=\mathcal{R}_{j}^{P}+\mathcal{R}_{j}^{M D}$, where the superscript $P$ denotes that this is the part of the disturbing function due to the giant planets only and the superscript $M D$ denotes that this is the part of the disturbing function due to the MD. They are expressed as:

$$
R_{j}^{P}=n_{j} a_{j}^{2}\left(\frac{1}{2} A_{j j}^{P} e_{j}^{2}+\sum_{k=1, k \neq j}^{N} A_{j k}^{P} e_{j} e_{k} \cos \left(\varpi_{j}-\varpi_{k}\right)\right.
$$$$
\left.+\frac{1}{2} B_{j j}^{P} I_{j}^{2}+\sum_{k=1, k \neq j}^{N} B_{j k}^{P} I_{j} I_{k} \cos \left(\Omega_{j}-\Omega_{k}\right)\right),
$$

$$
R_{j}^{M D}=n_{j} a_{j}^{2}\left(\frac{1}{2} A_{j j}^{M D} e_{j}^{2}+\frac{1}{2} B_{j j}^{M D} I_{j}^{2}\right),
$$

where $N$ is the number of giant planets, the coefficients $\left\{A_{j k}^{P}, 1 \leq j \leq N, 1 \leq\right.$ $k \leq N\}$ and $\left\{B_{j k}^{P}, 1 \leq j \leq N, 1 \leq k \leq N\right\}$ are frequencies associated to the apsidal and nodal precessions, respectively, of the giant planets without the MD and from which the eigenfrequencies $g_{i}^{P}$ and $f_{i}^{P}$, respectively describing the apsidal and nodal evolutions of the planetary system, are determined. The coefficients $A_{j j}^{M D}$ and $B_{j j}^{M D}$ are frequencies associated to the apsidal and nodal precessions of the $j$ th giant planet with the presence of the MD only. We can see that in equation 2, there are no coupling terms between the eccentricities of the giant planets and the "eccentricity" of the MD and between the inclinations of the giant planets and the "inclination" of the MD because of the axisymmetry and the planar symmetry of the MD. We can determine $\mathcal{R}_{j}^{P}$ analytically (see appendix A). To determine the MD contribution to the apsidal precession 
frequency $A_{j j}^{M D}$, we calculate the gravitational potential numerically, and we averaged it on one period of the orbit of the planet, keeping its inclination fixed to zero, for values of the eccentricity starting from zero until $e_{\max }=0.002$ and by increasing the eccentricity of 0.0001 at each step. We obtain the averaged gravitational potential as a function of the eccentricity, corresponding to the disk contribution of the apsidal part of the disturbing function which is of the form $\mathcal{R}_{j}^{M D}=\frac{1}{2} n_{j} a_{j}^{2} A_{j j}^{M D} e_{j}^{2}$ (see appendix A, figure 6, top panel). Second, we use the same method to determine the MD contribution to the nodal precession frequency $B_{j j}^{M D}$. We fix the eccentricity equal to zero and starting with an inclination equal to zero, we increase it with a step of $0.0001 \mathrm{rad}$ until it reaches the value $I_{\max }=0.002 \mathrm{rad}$. At each step, we calculate the gravitational potential averaged on one period of its orbit. We obtain the disk contribution to the nodal part of the disturbing function which is of the form $\mathcal{R}_{j}^{M D}=\frac{1}{2} n_{j} a_{j}^{2} B_{j j}^{M D} I_{j}^{2}$ (see appendix A, figure 6, bottom panel). Then, using a linear regression we obtain the values of the apsidal precession frequency $A_{j j}^{M D}$ and of the nodal precession frequency $B_{j j}^{M D}$. By using the frequencies $A_{j j}^{t o t}=A_{j j}^{P}+A_{j j}^{M D}$ and $B_{j j}^{t o t}=B_{j j}^{P}+B_{j j}^{M D}$, we can determine the eigenfrequencies $g_{i}^{\text {tot }}$ and $f_{i}^{\text {tot }}$ respectively describing the nodal and apsidal evolutions of the giant planets which account for the effect of the MD. To calculate the MD contribution to the free frequencies $A^{t o t}=A^{P}+A^{M D}$ and $B^{t o t}=B^{P}+B^{M D}$ of the apsidal and nodal free oscillations of a LD object, we can operate in the same way as previously for the giant planets. The apsidal and nodal secular resonances are localized at semi-major axes where $A^{\text {tot }}=g_{i}^{t o t}$ and $B^{\text {tot }}=f_{i}^{t o t}$ respectively.

\section{Positions of the nodal secular resonances in the primordial orbital configuration of the giant planets}

\subsection{Model}

In the first part, we look at the positions of the secular resonances in the initial conditions of the models proposed by Deienno et al. (2017). They studied the dynamical evolution of the giant planets, interacting with the MD, from several multiresonant configurations. In their models, there are initially Jupiter, Saturn, Uranus and Neptune as well as an extra ice giant. This additional planet is ejected during the dynamical instability. They found that only four 
multiresonant configurations can lead to the current orbital architecture of the outer solar system. These configurations are the following: (a) 3:2, 3:2, 4:3, $4: 3$; (b) $3: 2,3: 2,3: 2,3: 2$; (c) $3: 2,3: 2,2: 1,3: 2$; (d) $3: 2,3: 2,2: 1$, 2:1, with the semi-major axis of Jupiter initially at $\sim 5.4$ AU. We use those four multiresonant configurations. We place the semi-major axis of Jupiter at $5.4 \mathrm{AU}$, the inner edge of the MD at $1 \mathrm{AU}$ from Neptune and its outer edge at 30 AU from the Sun. The radial density profile of the MD is of the form $\Sigma(r)=\Sigma_{0} \frac{r_{0}}{r}$. Its vertical density profile follows a Gaussian law with $\sigma_{z}=r \tan \left(\sigma_{i}\right)$ where $\sigma_{i}=2^{\circ}$. We choose to fix the mass of the extra ice giant to $15 M_{\oplus}$ and we place it between Saturn and Uranus. The giant planets being in a chain of first order resonances, in their disturbing functions the apsidal and nodal parts of the resonant part contain terms of orders $\mathcal{O}(e)$ and $\mathcal{O}\left(e I^{2}\right)$ respectively whereas in the secular part, the apsidal and nodal terms are of orders $\mathcal{O}\left(e^{2}\right)$ and $\mathcal{O}\left(I^{2}\right)$. Thus, for the apsidal part, the resonant effects are stronger than the secular ones so the computation of the apsidal eigenfrequencies of the planetary system by using the linear secular theory would be wrong and it would not give the correct positions of the secular resonances. Hence, we cannot use the secular theory to look at the positions of the apsidal secular resonances. For the nodal part, the secular effects are more important than the resonant ones, so we are allowed to use the secular theory. However, for the configurations (c) and (d), some giant planets have a ratio between their semi-major axes corresponding to a 3:1 MMR. In such a resonance, the nodal part of the resonant part of the disturbing function is of order $\mathcal{O}\left(I^{2}\right)$ and it is as high as in the secular part. Depending on the libration or circulation of the resonant angles involved in this resonance, it can have the effect of slightly moving the positions of the secular resonances from the original ones.

\subsection{Positions of the nodal secular resonances}

In the secular theory, if we do not consider the MD but only the five giant planets, one of the eigenfrequencies $f_{i}$ associated to the inclinations and to the nodal precessions is null. The reason is that the choice of the reference plane being arbitrary, the inclination of a planet in regards to the reference plane evolves as a function of its inclination with respect to the other planets. In other words, there is an invariant plane, corresponding to the plane orthogonal 
to the total angular momentum vector of the planetary system (hereafter called the planetary plane), that does not precess because of the invariance of the total angular momentum. The frequency in question (usually denoted $f_{5}$ for the Solar System) is associated to the precession rate of the planetary plane relative to the arbitrary reference plane, and therefore it is zero. Now, if we add the MD, the total angular momentum of the giant planets is not conserved anymore and the planetary plane precesses with the frequency $f_{5}$ which is no longer equal to zero. However, the MD being static in our model, if we put the constraint that the orthogonal direction to the plane of symmetry of the MD is aligned with the total angular momentum of the planets, the amplitude of the oscillation corresponding to the $f_{5}$ frequency is null. Nevertheless, if the MD is in such a way that its orthogonal direction is misaligned with the total angular momentum of the planets, the amplitude associated to the $f_{5}$ frequency is not null anymore.

Figure 1 shows the positions of the nodal secular resonances for the orbital configuration $3: 2,3: 2,3: 2,3: 2$. The frequencies are negative because the nodal precessions are retrograde. We arbitrarily choose the subscripts of the eigenfrequencies according to their values: the lower is the absolute value of the frequency the higher is the subscript, except for the $f_{5}$ frequency corresponding to the frequency of the precession of the planetary plane of the giant planets. We represent three different cases: (a) in the first case, only the giant planets are present (top panel). All the secular resonances are located below $20 \mathrm{AU}$. (b) In the second case, we add the MD, with a mass $M_{d i s k}=40 M_{\oplus}$, in the model but it affects only the giant planets. It has for effect to increase the absolute values of the eigenfrequencies but the nodal free frequency of the LD object remains the same as in the case where only the giant planets are present (bottom panel, the horizontal lines represent the values of the eigenfrequencies of the planetary system affected by the MD and the dashed curve represents the nodal free frequency of the LD object not affected by the MD). It corresponds to the case of numerical integrations where the MD is represented by a set of massive planetesimals acting on the planets but not on the other planetesimals. The $f_{6}, f_{7}, f_{8}$ and $f_{9}$ secular resonances are still located below 20 AU. However, the secular resonance associated to the $f_{5}$ frequency, which is not null anymore, 
is located in the LD near $33 \mathrm{AU}$. (c) Finally, in the third case, the MD also affects the LD objects and increases their nodal free frequencies (bottom panel, the full black curve represents the nodal free frequency of the LD object affected by the MD). The positions of the $f_{6}$ and $f_{7}$ secular resonances remain nearly the same as in the other two cases but the $f_{8}$ and $f_{9}$ secular resonances are pushed toward $\sim 30 \mathrm{AU}$ and the $f_{5}$ secular resonance is located near $44 \mathrm{AU}$ which is inside the region of the current cold classical Kuiper Belt. This highlights that the consideration of the direct effect of the MD on the LD objects shifts significantly the positions of three secular resonances. The fact that a secular resonance was primordially located in the region corresponding to the current classical Kuiper Belt, which is a stable region populated with objects whose orbits only have had a slight evolution, is important because it can let a dynamical signature which can be observable today. The migration of the giant planets, the evolution of the shape of the disk and its loss of mass, cause the migration of the secular resonances. If the excitation timescale of the $f_{5}$ secular resonance is short enough with respect to its migration timescale, the resonance can remove the cold population objects from the swept region by rising their inclinations to values higher than $5^{\circ}$, which roughly corresponds to the inclination border beyond which the objects are not considered as cold anymore, and it creates a local population of objects with excited inclinations but with eccentricities staying low, which are on stable orbits. Otherwise, if the excitation timescale of the $f_{5}$ secular resonance is too long compared with its migration timescale, its passage preserves the cold population while slightly exciting it. In this configuration, the $f_{8}$ and $f_{9}$ secular resonances are located just below 30 AU whereas the 2:1 MMR with Neptune is located near 26 AU. Thus, the two secular resonances must excite the inclinations of the objects before the passage of the sweeping 2:1 MMR during the migration of Neptune. This could have affected the inclination distribution of the objects captured by the 2:1 MMR.

Hence, we will now look at the positions of the $f_{5}$ secular resonance in the different orbital configurations proposed by Deienno et al. (2017) and with several masses of the MD. The results are shown in table 1 . The $f_{5}$ secular resonance is located in the current cold Kuiper Belt for the configuration 3:2, $3: 2,4: 3,4: 3$ with $M_{d i s k}=20 M_{\oplus}$ and for the configuration $3: 2,3: 2,3: 2,3: 2$ for 

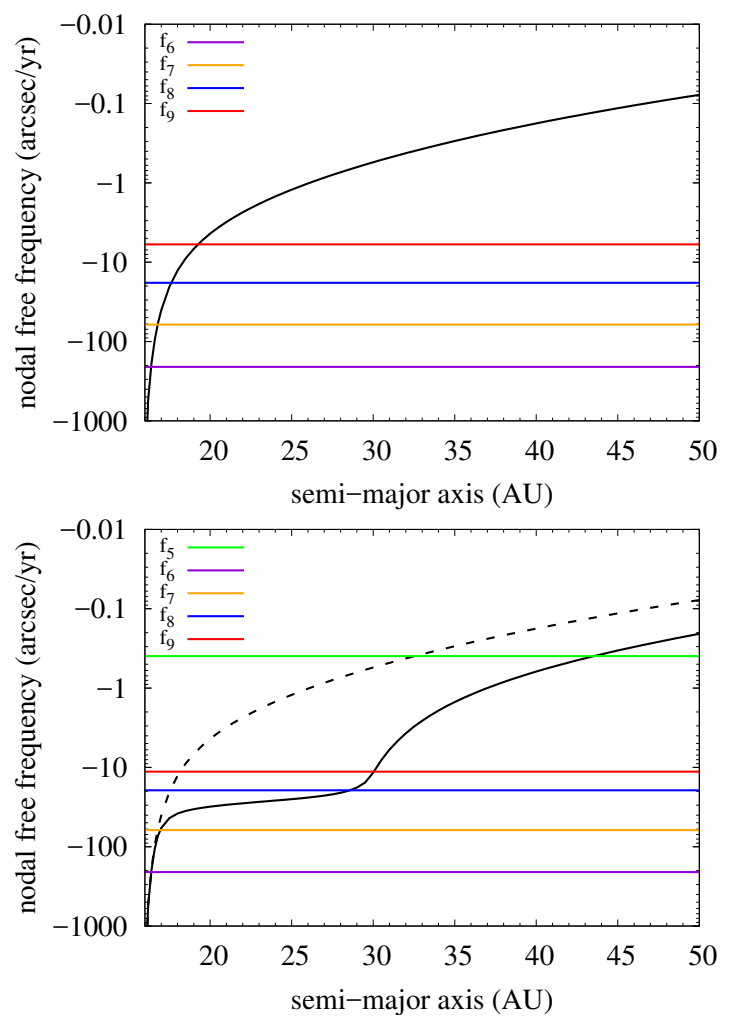

Figure 1: Nodal free frequency of a LD object as a function of its semi-major axis in the configuration $3: 2,3: 2,3: 2,3: 2$, in a model without the MD (top panel) and in a model with a $\mathrm{MD}$ of mass $M_{d i s k}=40 M_{\oplus}$ (bottom panel). The horizontal lines are the nodal eigenfrequencies. In the bottom panel, the dashed curve is the nodal free frequency in the case where the MD does not act on the LD object and the full black curve is the nodal free frequency but in the case where the MD acts on the LD object. The nodal secular resonances occur at the semi-major axes where the nodal free frequency crosses one of the nodal eigenfrequencies.

the three different masses of the disk. In the configuration 3:2, 3:2, 2:1, 3:2, with $M_{d i s k}=40 M_{\oplus}$ and $M_{d i s k}=60 M_{\oplus}$, the resonance is located at the outer border of the current cold Kuiper Belt, near the 2:1 MMR. With $M_{\text {disk }}=20 M_{\oplus}$, it is located in a region where an extension of the cold population beyond the 2:1 MMR has been discovered (Bannister et al., 2018). In the configuration $3: 2,3: 2,2: 1,2: 1$, the resonance is located in a region where cold Kuiper Belt objects have still not been observed so far. Finally, in the configuration 3:2, 3:2, 4:3, 4:3, with $M_{d i s k}=40 M_{\oplus}$ and $M_{d i s k}=60 M_{\oplus}$ the resonance is located in a region where the current cold Kuiper Belt does not exist anymore because the 


\begin{tabular}{|c|c|c|}
\hline Orbital configuration & $M_{\text {disk }}\left(M_{\oplus}\right)$ & Position of $f_{5}(\mathrm{AU})$ \\
\hline \multirow{3}{*}{$3: 2,3: 2,4: 3,4: 3$} & 20 & 42.7 \\
& 40 & 41.0 \\
& 60 & 40.5 \\
\hline $3: 2,3: 2,3: 2,3: 2$ & 20 & 45.0 \\
& 40 & 43.5 \\
& 60 & 43.3 \\
\hline $3: 2,3: 2,2: 1,3: 2$ & 20 & 48.3 \\
& 40 & 47.5 \\
& 60 & 47.7 \\
\hline $3: 2,3: 2,2: 1,2: 1$ & 20 & 55.1 \\
& 40 & 54.7 \\
& 60 & 54.7 \\
\hline
\end{tabular}

Table 1: Position of the $f_{5}$ secular resonance for four orbital configurations and for several masses of the MD.

\subsection{Efficiency of the $f_{5}$ secular resonance}

We performed simple numerical integrations in order to illustrate the efficiency of the $f_{5}$ secular resonance. We cannot include directly the massive disk and compute the forces it exerts on the bodies because it is computationally too heavy. Therefore, we only include the nodal precession it exerts. To do so, we proceed as follow: as a first step, for a given shape and mass of the disk, we use the method described in section 2 in order to sample the values of the nodal precession frequency as a function of the semi-major axis. We sample the frequency every $0.1 \mathrm{AU}$ in a range from $1 \mathrm{AU}$ to $60 \mathrm{AU}$. It is illustrated in figure 2. Then, we use a linear interpolation in order to have a continuous function of the frequency. In a second step, to allow the variation of the longitude of 
the node of a body, we use the method of Lee and Peale (2002) implemented in the same way as Wolff et al. (2012) in the Mercury6 N-body integrator package (Chambers, 1999), adding acceleration and velocity perturbation terms corresponding to the variation of the longitude of the node, as described in appendix B.

Here, the goal of our numerical integrations is not to include the effect of the $f_{5}$ secular resonance in a complete model of dynamical evolution of the giant planets and of the Kuiper Belt and then to compare the Kuiper Belt obtained with the observations. It would demand to construct a strong model where the evolution of the shape and of the mass of the disk is coherent with the migration of the giant planets. The construction of such a model is beyond the scope of this study and is left for future work. The aim of the present study is qualitative and is to illustrate how efficient the $f_{5}$ secular resonance can be in a numerical integration. Therefore, in the present model, the giant planets remain unrealistically at their initial semi-major axes during the whole numerical integration and the shape of the MD does not evolve but we allow its mass to decrease exponentially with time, simulating the migration of the $f_{5}$ secular resonance. We do not allow the giant planets to migrate because, as we can see in figure 2 , there is a strong variation of the nodal precession frequency in the region of the MD. However, as Neptune follows its outward migration, the inner border of the MD moves back and Neptune never reaches the region where this strong variation takes place. So, if we allow Neptune to migrate but we do not adapt the shape of the disk to it, the nodal precession frequency of Neptune would be more enhanced than it is in a more realistic model. We do not explore the effect of the $f_{8}$ and $f_{9}$ secular resonances either because the decaying disk would cause them to move inward whereas the migration of the giant planets would make the resonances to migrate outward. Therefore, if the effect of the outward migration is stronger than that of the inward migration, a model that accounts for the two effects would see the outward migration of the resonances whereas in our model we only see an inward migration, thus the representation would be wrong.

We illustrate our numerical integrations with the example of the multiresonant configuration $3: 2,3: 2,2: 1,3: 2$ which is evaluated as the most probable 
by Deienno et al. (2017). We choose the initial mass of the massive disk to be $M_{d i s k}=20 M_{\oplus}$. We use fictitious forces of Lee and Peale (2002) to keep the semi-major axes of the giant planets in the multiresonant configuration and to damp their eccentricities. Given that the efficiency of the $f_{5}$ secular resonance depends on the inclination of the planetary plane with respect to the mean plane of the disk, we did numerical integrations with different initial inclinations of the planetary plane. We place the giant planets initially on coplanar orbits. We put massless particles from $45 \mathrm{AU}$ to $55 \mathrm{AU}$, with one particle every 0.1 AU. We generate the eccentricities and the inclinations of the particles with a Rayleigh distribution with a mode of 0.01 and 0.01 radians respectively. The other angles of the giant planets and of the particles are uniformly distributed over $360^{\circ}$. Given that in the linear secular theory, the precession frequency is linearly proportional to the mass of the disk, we can account for the depletion of the disk directly by modifying the precession frequency. We represent the depletion of the mass of the disk by an exponential decay with a timescale $\tau$. In a first set of numerical integrations, we explore the effect of the inclination of the planetary plane. We choose a disk depletion timescale $\tau=50 \mathrm{Myr}$ and we run three numerical integrations over 10 Myr with a timestep of 100 days and with three different initial inclinations of the planetary plane: $0^{\circ}, 1^{\circ}$ and $2^{\circ}$. Figure 3 presents the results and shows that the higher the inclination of the planetary plane, the higher the efficiency of the $f_{5}$ secular resonance.

In a second set of numerical integrations, we fix the initial inclination of the planetary plane at $1^{\circ}$ but we try three values of the depletion timescale: $\tau=50$ Myr, $\tau=30$ Myr and $\tau=10$ Myr. We run our numerical integrations for 200 Myr. The time step is kept at 100 days. Figure 4 shows the results, with the semi-major axes averaged over the last $50 \mathrm{Myr}$ and the proper inclinations. In the case $\tau=50 \mathrm{Myr}$ the resonance sweeps from $\sim 48$ AU to $\sim 55 \mathrm{AU}$ in $\sim 100 \mathrm{Myr}$. The rising of the proper inclinations after $54 \mathrm{AU}$ is associated to a dephasing effect. In the case $\tau=30$ Myr it sweeps in $\sim 60$ Myr and for $\tau=10$ Myr it sweeps in $\sim 30$ Myr.

We remind that those numerical integrations are not autocoherent and it would demand to do stronger effort in order to perform numerical integrations including the effect of the massive disk with the latter evolving with respect to 
the migration of Neptune in a consistent way. Besides, there is an uncertainty of the time of the triggering of the instability. It has a consequence on the timescale during which the $f_{5}$ secular resonance is efficient. During the preinstability period, the mass of the disk is slowly depleted and remains high enough, so the resonance slowly moves from its initial location. Then, during the instability, the mass of the disk is quickly depleted and the secular resonance migrates very rapidly toward higher semi-major axes.

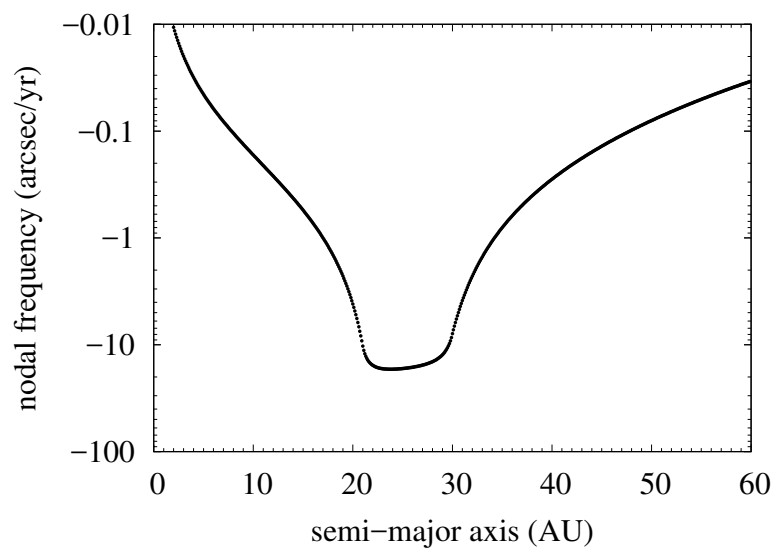

Figure 2: The frequency of the nodal precession caused by the MD as a function of the semimajor axis. The mass of the MD is $M_{d i s k}=20 M_{\oplus}$, its inner edge is at $21 \mathrm{AU}$ and its outer edge at $30 \mathrm{AU}$.

\section{Positions of the secular resonances just before the planetary insta- bility}

During the migration of the giant planets, they interact with the planetesimals of the MD and some of the latter get ejected. Therefore, the MD is gradually depleted. The aim of this section is to check whether the shift of the secular resonances remains important or not and to check the position of the $f_{5}$ secular resonance, just before the planetary instability. For that purpose, we use the results of a numerical integration for a Neptune migration scenario developed by Nesvorný (2015a). It also includes the planetary instability, represented via a jumping Neptune, which occurred when the ice giant is near 28 AU in order to explain the kernel in the cold population (Nesvorný, 2015b). 


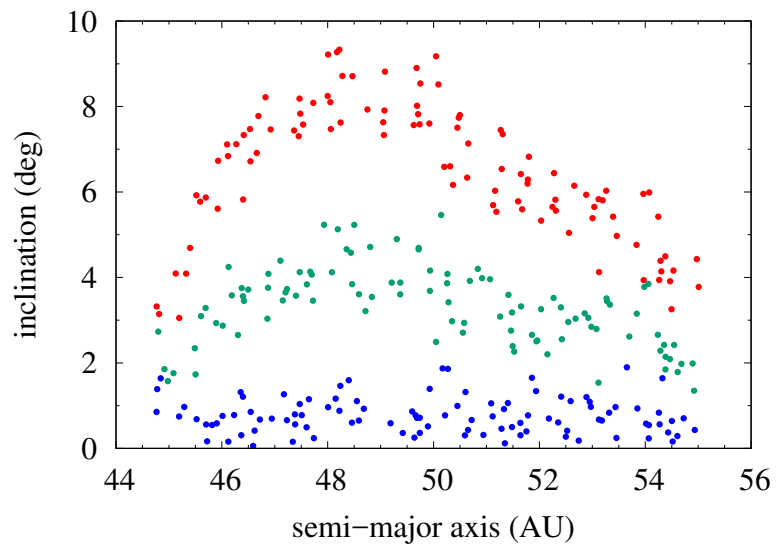

Figure 3: Inclinations of the particles after $10 \mathrm{Myr}$. The multiresonant configuration of the giant planets is $3: 2,3: 2,2: 1,3: 2$. The initial mass of the MD is $M_{d i s k}=20 M_{\oplus}$, its inner edge is at $21 \mathrm{AU}$, its outer edge at $30 \mathrm{AU}$ and its depletion timescale is $50 \mathrm{Myr}$. The three colors of the particles represent the results of three different numerical integrations, for which the initial inclination of the planetary plane with respect to the mean plane of the disk is $i=0^{\circ}$ (blue dots), $i=1^{\circ}$ (green dots) and $i=2^{\circ}($ red dots $)$.

\footnotetext{
The context of this numerical integration is different than that of numerical integrations of Deienno et al. (2017). The aim of the study of Nesvorný (2015a) was to reproduce the inclination distribution of the hot Kuiper Belt population from a set of massless particles initially located between Neptune and 30 AU with a slow radial migration of Neptune. Here, there are only the four giant planets and they are not in a multiresonant configuration anymore. Thus, it is possible to look at the positions of the apsidal secular resonances. The initial positions of Jupiter, Saturn and Uranus correspond to their current ones and Neptune was placed with an initial semi-major axis lower than 25 AU. Its radial migration and its eccentricity and inclination damping were driven by fictitious forces.
}

\subsection{Model}

From the orbital elements of the particles provided by the numerical integration of Nesvorný (2015a) we estimate the distribution of the MD at $t=3$ Myr before the instability between the giant planets. Let $(r, \theta, z)$ be the heliocentric coordinates. We assume that the MD distribution is axisymmetric and 


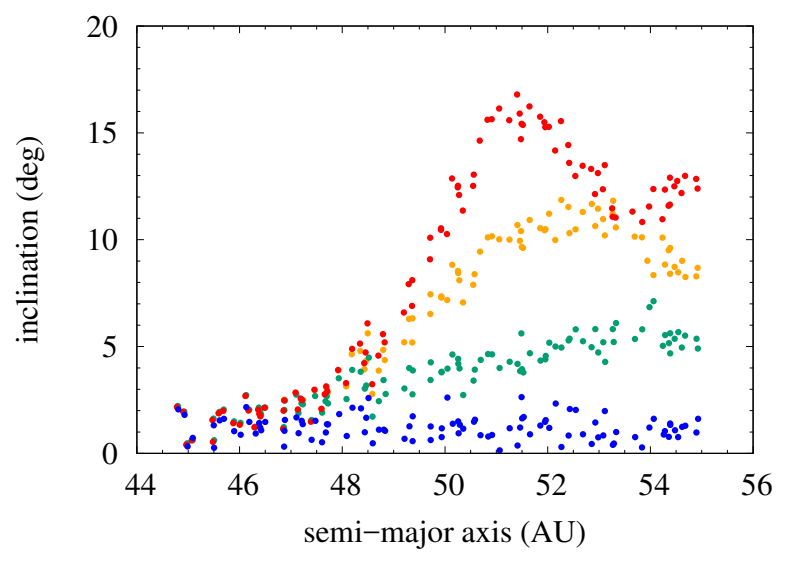

Figure 4: Proper inclinations of the particles after $200 \mathrm{Myr}$ numerical integrations. The multiresonant configuration of the giant planets is $3: 2,3: 2,2: 1,3: 2$ and the initial inclination of the planetary plane with respect to the mean plane of the disk is $i=1^{\circ}$. The initial mass of the MD is $M_{\text {disk }}=20 M_{\oplus}$, its inner edge is at $21 \mathrm{AU}$ and its outer edge at $30 \mathrm{AU}$. The blue dots represent the results of the particles for a numerical integration where the nodal precession due to the MD is not included. The other colors represent the results for numerical integrations whith different depletion timescales $\tau$ of the mass of the MD. The green dots are for $\tau=10 \mathrm{Myr}$, the orange dots for $\tau=30 \mathrm{Myr}$ and the red dots for $\tau=50 \mathrm{Myr}$. The semi-major axes are averaged over the last $50 \mathrm{Myr}$ of the numerical integrations.

uncoupled in $r$ and $z$. We construct histograms of the $r$ and $z$ distributions from which we obtain the following density profiles:

439

$$
\sigma_{r}(r)= \begin{cases}\frac{23.5}{2 \pi} & \text { if } r \leq 28 \mathrm{AU}, \\ \frac{2961}{\pi r(r-19)} & \text { if } 28 \mathrm{AU}<r \leq 1000 \mathrm{AU}, \\ 0 & \text { if } r>1000 \mathrm{AU},\end{cases}
$$

440 and

$$
\lambda_{z}(z)= \begin{cases}\exp \left(-\frac{|z|}{10}\right) & \text { if }-100 \mathrm{AU} \leq z \leq 100 \mathrm{AU} \\ 0 & \text { otherwise }\end{cases}
$$

442 where $r$ and $z$ are expressed in $\mathrm{AU}, \sigma_{r}(r)$ is the radial surface density in $\mathrm{kg} . \mathrm{AU}^{-2}$

${ }_{443}$ and $\lambda_{z}(z)$ is the vertical linear density in $\mathrm{kg} . \mathrm{AU}^{-1}$. Here, those two last quan- 


\begin{tabular}{|c|c|c|}
\cline { 2 - 3 } \multicolumn{1}{c|}{} & \multicolumn{2}{c|}{ Orbital elements } \\
\cline { 2 - 3 } \multicolumn{1}{c|}{} & $\mathrm{a}(\mathrm{AU})$ & $\mathrm{e}$ \\
\hline Jupiter & 5.201731 & 0.01824838 \\
\hline Saturn & 9.578610 & 0.05086522 \\
\hline Uranus & 18.16524 & 0.03557539 \\
\hline Neptune & 27.80457 & 0.01387243 \\
\hline
\end{tabular}

Table 2: Semi-major axes and eccentricities of the giant planets 3 Myr before the planetary instability.

tities are not normalized. The density profile of the MD is:

$$
\rho(r, z)=M_{d i s k} \frac{\sigma_{r}(r) \lambda_{z}(z)}{\int_{r=0}^{r=R} \int_{z=z_{\min }}^{z=z_{\max }} \int_{\theta=0}^{\theta=2 \pi} \sigma_{r}(r) \lambda_{z}(z) r \mathrm{~d} r \mathrm{~d} z \mathrm{~d} \theta},
$$

in $\mathrm{kg} \cdot \mathrm{AU}^{-3}$, where the denominator is used to normalize the function. The mass of the MD is chosen with an initial value of $20 M_{\oplus}$, because the migration timescale of Neptune in that numerical integration is typical of that with such a mass. It has the value $5.9604 M_{\oplus}$ at the time $t=3$ Myr before the planetary instability.

\subsection{Results}

We apply the method described in section 2 to evaluate the apsidal and nodal eigenfrequencies of the planetary system and the free frequencies of the LD object.

Table 2 shows the semi-major axes and eccentricities of the giant planets 3 Myr before the planetary instability. The $f_{5}$ secular resonance is located beyond $150 \mathrm{AU}$, because the mass of the MD is low and the radial distance of the $f_{5}$ secular resonance increases as the disk loses mass.

Figure 5 presents the results that we obtained for the positions of the $g_{8}$ secular resonance, exhibited by the dashed vertical lines, according to several perspectives. $A^{P}$ (red curve) and $g_{8}^{P}$ (red dashed horizontal line) are the frequencies in the case where the apsidal precessions are only driven by the giant planets, whereas $A^{\text {tot }}$ (blue curve) and $g_{8}^{\text {tot }}$ (blue dashed horizontal line) are the 
frequencies in the case where the apsidal precessions are driven by the giant planets but also by the MD. The red dashed vertical line shows the position of the resonance in the case where the MD does not act neither on the giant planets nor the LD objects and then is located where $A^{P}=g_{8}^{P}$, at $37.21 \mathrm{AU}$. The purple dashed vertical line corresponds to the case where the MD acts on the giant planets but not on the LD objects and is located where $A^{P}=g_{8}^{t o t}$, at 37.59 AU. Finally, the blue vertical line is the ideal case where the MD acts both on the giant planets and on the LD objects. It is located where $A^{\text {tot }}=g_{8}^{\text {tot }}$, at $37.44 \mathrm{AU}$. It makes a shift of $0.6 \%$ compared to the case where the MD is neglected. The other resonances are also shifted by less than $1 \%$. We conclude that the shifts imposed by the MD are negligible.

We also check the positions of the secular resonances 50 Myr after the planetary instability. In this case, the mass of the disk is $2.9006 M_{\oplus}$, two times lower than at 3 Myr before the instability, but the peak of the mass distribution is located at $\sim 30 \mathrm{AU}$, which is closer from the Kuiper Belt than in the previous case. However, we find that the $f_{5}$ secular resonance remains located beyond $150 \mathrm{AU}$ and the shift of the other secular resonances is even smaller than in the previous case.

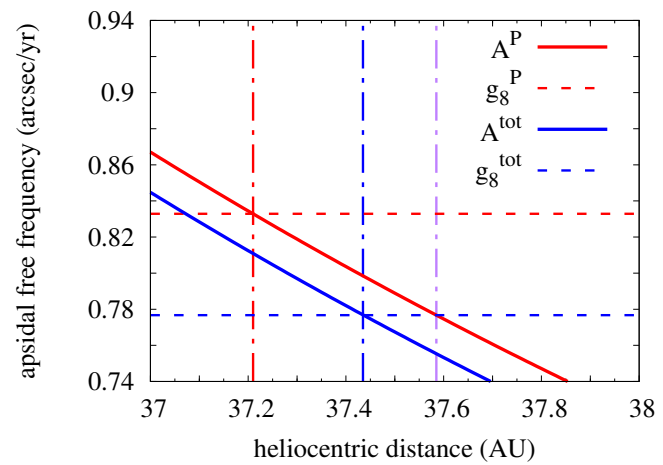

Figure 5: Position of the $g_{8}$ secular resonance in the case without the MD (red curve) and with it (blue curve), 3 Myr before the instability between the giant planets. Horizontal dashed lines represent the value of $g_{8}$. We exhibit the intersection of the curves with the horizontal dashed lines by dashed vertical lines which is the position of the $g_{8}$ secular resonance. The purple dashed vertical line shows the position of $g_{8}$ in the case where the MD acts on the giant planets but not on the LD object. 


\section{Discussion and conclusion}

Studies of the primordial dynamical evolution of the giant planets and of the primordial planetesimal disk have shown that the latter must have a sharp edge near $30 \mathrm{AU}$ in its mass distribution. The part of the disk beyond this sharp edge is a light extension of the disk so as to make unefficient the planetesimal-driven migration of Neptune beyond this edge and to stop the ice giant at its current position. Besides, current models starting with initial conditions presenting a continuous mass distribution in the planetesimal disk between Neptune and 50 AU do not succeed in sufficiently depleting the mass of the cold Kuiper Belt region. The cold Kuiper Belt population is supposed to be formed from this light extension.

That being said, dynamical studies of the objects of the light part of the disk have so far always been restricted to the dynamical evolution of those objects under the effect of the gravitational interactions with the giant planets only. However, given the important mass of the disk between Neptune and $30 \mathrm{AU}$ $\left(\sim 10-60 M_{\oplus}\right)$, it can have a non-negligible influence. This is what we wanted to check in this paper. We have called the massive disk (MD) the part of the planetesimal disk between Neptune and 30 AU, and the light disk (LD) the part of the planetesimal disk beyond 30 AU. We have studied the different positions that the secular resonances can have in three different situations: (a) a case where the MD is not included, (b) a case where the MD modifies the apsidal and nodal precessions of the giant planets but not those of the LD objects and (c) a case where the MD also acts on the apsidal and nodal precessions of the LD objects.

In section 3, we have investigated on the positions of the secular resonances once the solar nebula vanished and the giant planets were still locked in a multiresonant configuration. We could only look at the positions of the nodal secular resonances because the resonant dynamics is dominant in the evolution of the longitudes of the perihelion of the giant planets. We have found that the $f_{5}$ secular resonance, driven by the precession of the total angular momentum of the giant planets, is located in the region of the LD in several configurations. It can have an effect in exciting the inclinations of the LD objects. Consequently, it can create a population of objects with high inclinations but low eccentricities. 
Its efficiency in rising their inclinations mainly depends on the angle between the planetary and the MD planes. While revising this manuscript we became aware of the work of Toliou and Tsiganis (2019), performed independently, that find results similar to ours on the existence and the role of a non-zero $f_{5}$ frequency during the disk phase.

In planetary formation models, the planets and the planetesimal disk formed from the same protoplanetary disk in the plane of the solar equator. In the current Solar System, we observe an inclination of $\sim 6^{\circ}$ between the solar equator and the invariant plane. It has been shown that the presence of a ninth planet located in the outer Solar System can explain this tilt (Bailey et al. (2016), Gomes et al. (2017) and Lai (2016)). Another possibility, as suggested by Batygin (2012), is that the tilt can be produced by the passage of a star in the birth cluster. The passage of a star can warp the protoplanetary disk, making its outer part inclined with respect to the inner part where the planets are formed. Moreover, Izidoro et al. (2015) have shown that during the accretion of Uranus and Neptune, ejections of planetary embryos caused by close encounters were common. As ejected objects take away with them a part of the total angular momentum, the ones remaining in the Solar System have an angular momentum different from the total one and that is not conserved. Therefore, the orientation of the plane orthogonal to the angular momentum of the remaining objects varies with time, until the Solar System reaches its current architecture and the ejections of objects stopped, leaving what is currently called the invariant plane. All of these elements give weight to the conception that the invariant plane of the planets got misaligned with the plane of the disk.

In section 4, we have looked at the positions of the secular resonances during the migration of Neptune just before the instability between the giant planets occurred, when Neptune is near 28 AU, from data provided by the works of Nesvorný (2015a). We have found that during this phase of the planetary evolution, the mass of the MD is not high enough anymore and that consequently the positions of the secular resonances are only shifted by less than $1 \%$ with respect to the case where the MD is neglected. In this case, if numerical integrations omit the effects of the MD, the evolution of the LD objects will be qualitatively the same as if they do not. 
We thank the reviewers for helpful comments that improved the manuscript. We acknowledge support by the French ANR, project number ANR-13-13-BS050003-01 project MOJO (Modeling the Origin of JOvian planets). We acknowledge David Nesvorný for providing us with data of a numerical integration.

\section{References}

Bailey, E., Batygin, K., Brown, M.E., 2016. Solar Obliquity Induced by Planet Nine. AJ 152, 126. doi:10.3847/0004-6256/152/5/126, arXiv:1607.03963.

Bannister, M.T., Gladman, B.J., Kavelaars, J.J., Petit, J.M., Volk, K., Chen, Y.T., Alexandersen, M., Gwyn, S.D.J., Schwamb, M.E., Ashton, E., Benecchi, S.D., Cabral, N., Dawson, R.I., Delsanti, A., Fraser, W.C., Granvik, M., Greenstreet, S., Guilbert-Lepoutre, A., Ip, W.H., Jakubik, M., Jones, R.L., Kaib, N.A., Lacerda, P., Van Laerhoven, C., Lawler, S., Lehner, M.J., Lin, H.W., Lykawka, P.S., Marsset, M., Murray-Clay, R., Pike, R.E., Rousselot, P., Shankman, C., Thirouin, A., Vernazza, P., Wang, S.Y., 2018. OSSOS. VII. 800+ Trans-Neptunian Objects-The Complete Data Release. ApJS 236, 18. doi:10.3847/1538-4365/aab77a, arXiv:1805.11740.

Batygin, K., 2012. A primordial origin for misalignments between stellar spin axes and planetary orbits. Nature 491, 418-420. doi:10.1038/nature11560.

Batygin, K., Brown, M.E., Fraser, W.C., 2011. Retention of a Primordial Cold Classical Kuiper Belt in an Instability-Driven Model of Solar System Formation. ApJ 738, 13. doi:10.1088/0004-637X/738/1/13, arXiv:1106.0937.

Chambers, J.E., 1999. A hybrid symplectic integrator that permits close encounters between massive bodies. MNRAS 304, 793-799. doi:10.1046/j. 1365-8711.1999.02379.x.

Dawson, R.I., Murray-Clay, R., 2012. Neptune's Wild Days: Constraints from the Eccentricity Distribution of the Classical Kuiper Belt. ApJ 750, 43. doi:10.1088/0004-637X/750/1/43, arXiv:1202.6060.

Deienno, R., Morbidelli, A., Gomes, R.S., Nesvorný, D., 2017. Constraining the Giant Planets' Initial Configuration from Their Evolution: Implications 
for the Timing of the Planetary Instability. AJ 153, 153. doi:10.3847/ 1538-3881/aa5eaa, arXiv: 1702. 02094

Fukushima, T., 2016. Numerical computation of gravitational field for general axisymmetric objects. MNRAS 462, 2138-2176. doi:10.1093/mnras/ stw1765.

Gomes, R., Deienno, R., Morbidelli, A., 2017. The Inclination of the Planetary System Relative to the Solar Equator May Be Explained by the Presence of Planet 9. AJ 153, 27. doi:10.3847/1538-3881/153/1/27, arXiv: 1607.05111.

Gomes, R., Levison, H.F., Tsiganis, K., Morbidelli, A., 2005. Origin of the cataclysmic Late Heavy Bombardment period of the terrestrial planets. Nature 435, 466-469. doi:10.1038/nature03676.

Gomes, R., Nesvorný, D., Morbidelli, A., Deienno, R., Nogueira, E., 2018. Checking the compatibility of the cold Kuiper belt with a planetary instability migration model. Icarus 306, 319-327. doi:10.1016/j .icarus. 2017.10.018, arXiv: 1710.05178.

Gomes, R.S., 2003. The origin of the Kuiper Belt high-inclination population. Icarus 161, 404-418. doi:10.1016/S0019-1035(02) 00056-8.

Gomes, R.S., Morbidelli, A., Levison, H.F., 2004. Planetary migration in a planetesimal disk: why did Neptune stop at 30 AU? Icarus 170, 492-507. doi:10.1016/j.icarus . 2004.03.011.

Haisch, Jr., K.E., Lada, E.A., Lada, C.J., 2001. Disk Frequencies and Lifetimes in Young Clusters. ApJL 553, L153-L156. doi:10.1086/320685, arXiv: astro-ph/0104347.

Hayashi, C., 1981. Structure of the Solar Nebula, Growth and Decay of Magnetic Fields and Effects of Magnetic and Turbulent Viscosities on the Nebula. Progress of Theoretical Physics Supplement 70, 35-53. doi:10.1143/PTPS. 70.35 .

Izidoro, A., Morbidelli, A., Raymond, S.N., Hersant, F., Pierens, A., 2015. Accretion of Uranus and Neptune from inward-migrating planetary embryos 
blocked by Jupiter and Saturn. A\&A 582, A99. doi:10.1051/0004-6361/ 201425525, arXiv:1506.03029.

Lai, D., 2016. Solar Obliquity Induced by Planet Nine: Simple Calculation. AJ 152, 215. doi:10.3847/0004-6256/152/6/215, arXiv:1608.01421.

Lee, M.H., Peale, S.J., 2002. Dynamics and Origin of the 2:1 Orbital Resonances of the GJ 876 Planets. ApJ 567, 596-609. doi:10.1086/338504.

Levison, H.F., Morbidelli, A., Van Laerhoven, C., Gomes, R., Tsiganis, K., 2008. Origin of the structure of the Kuiper belt during a dynamical instability in the orbits of Uranus and Neptune. Icarus 196, 258-273. doi:10.1016/j.icarus . 2007.11.035, arXiv:0712.0553.

Li, J., Zhou, L.Y., Sun, Y.S., 2008. The Effect of Sweeping Secular Resonances on the Classical Kuiper Belt. ChA\&A 32, 409-422. doi:10.1016/j . chinastron.2008.10.003.

Mamajek, E.E., 2009. Initial Conditions of Planet Formation: Lifetimes of Primordial Disks, in: Usuda, T., Tamura, M., Ishii, M. (Eds.), American Institute of Physics Conference Series, pp. 3-10. doi:10.1063/1.3215910, arXiv:0906.5011.

Masset, F., Snellgrove, M., 2001. Reversing type II migration: resonance trapping of a lighter giant protoplanet. MNRAS 320, L55-L59. doi:10.1046/j . 1365-8711.2001.04159.x, arXiv:astro-ph/0003421.

Morbidelli, A., Levison, H.F., Tsiganis, K., Gomes, R., 2005. Chaotic capture of Jupiter's Trojan asteroids in the early Solar System. Nature 435, 462-465. doi:10.1038/nature03540.

Morbidelli, A., Tsiganis, K., Crida, A., Levison, H.F., Gomes, R., 2007. Dynamics of the Giant Planets of the Solar System in the Gaseous Protoplanetary Disk and Their Relationship to the Current Orbital Architecture. AJ 134, 1790-1798. doi:10.1086/521705, arXiv:0706.1713.

Murray, C.D., Dermott, S.F., 2000. Solar System Dynamics. 
Nagasawa, M., Ida, S., 2000. Sweeping Secular Resonances in the Kuiper Belt Caused by Depletion of the Solar Nebula. AJ 120, 3311-3322. doi:10.1086/ 316856.

Nesvorný, D., 2015a. Evidence for Slow Migration of Neptune from the Inclination Distribution of Kuiper Belt Objects. AJ 150, 73. doi:10.1088/ 0004-6256/150/3/73, arXiv: 1504.06021

Nesvorný, D., 2015b. Jumping Neptune Can Explain the Kuiper Belt Kernel. AJ 150, 68. doi:10.1088/0004-6256/150/3/68, arXiv:1506.06019.

Nesvorný, D., 2018. Dynamical Evolution of the Early Solar System. ARA\&A 56, 137-174. doi:10.1146/annurev-astro-081817-052028, arXiv: 1807.06647.

Nesvorný, D., Vokrouhlický, D., 2016. Neptune's Orbital Migration Was Grainy, Not Smooth. ApJ 825, 94. doi:10.3847/0004-637X/825/2/94, arXiv: 1602.06988.

Toliou, A., Tsiganis, K., 2019. Secular resonance sweeping and orbital excitation in decaying disk. Celestial Mechanics and Dynamical Astronomy (submitted)

Tsiganis, K., Gomes, R., Morbidelli, A., Levison, H.F., 2005. Origin of the orbital architecture of the giant planets of the Solar System. Nature 435, 459-461. doi:10.1038/nature03539.

Wolff, S., Dawson, R.I., Murray-Clay, R.A., 2012. Neptune on Tiptoes: Dynamical Histories that Preserve the Cold Classical Kuiper Belt. ApJ 746, 171. doi:10.1088/0004-637X/746/2/171, arXiv:1112.1954.

\section{Appendix A}

In a planetary system with $N$ giant planets, the secular part of the disturbing functions $R_{j}^{P}$ of the $j$ th giant planet and $R^{P}$ of a LD object are (Murray and 
664

$$
R_{j}^{P}=n_{j} a_{j}^{2}\left(\frac{1}{2} A_{j j}^{P} e_{j}^{2}+\sum_{k=1, k \neq j}^{N} A_{j k}^{P} e_{j} e_{k} \cos \left(\varpi_{j}-\varpi_{k}\right)\right.
$$

$$
\left.+\frac{1}{2} B_{j j}^{P} I_{j}^{2}+\sum_{k=1, k \neq j}^{N} B_{j k}^{P} I_{j} I_{k} \cos \left(\Omega_{j}-\Omega_{k}\right)\right),
$$

666

673

675

674

$$
\begin{aligned}
R^{P}= & n a^{2}\left(\frac{1}{2} A^{P} e^{2}+\sum_{j=1}^{N} A_{j}^{P} e e_{j} \cos \left(\varpi-\varpi_{j}\right)\right. \\
& \left.+\frac{1}{2} B^{P} I^{2}+\sum_{j=1}^{N} B_{j}^{P} I I_{j} \cos \left(\Omega-\Omega_{j}\right)\right),
\end{aligned}
$$

where the terms with the superscript $P$ denotes that they are due to the planetary system only and the variables with the subscript $j$ and without it respectively relate to the $j$ th giant planet and to the LD object. $n$ is the mean motion, $a$ the semi-major axis, $e$ the eccentricity, $I$ the inclination, $\varpi$ the longitude of the perihelion and $\Omega$ the longitude of the node. The coefficients $A_{j j}^{P}, A_{j k}^{P}, B_{j j}^{P}$, $B_{j k}^{P}, A^{P}, A_{j}^{P}, B^{P}$ and $B_{j}^{P}$ are frequencies, which depend on the masses and semi-major axes of the bodies such that:

$$
\begin{aligned}
& A_{j j}^{P}=\frac{1}{4} n_{j} \sum_{k=1, k \neq j}^{N} \frac{m_{k}}{m_{c}+m_{j}} \alpha_{j k} \bar{\alpha}_{j k} b_{3 / 2}^{(1)}\left(\alpha_{j k}\right), \\
& A_{j k}^{P}=-\frac{1}{4} n_{j} \frac{m_{k}}{m_{c}+m_{j}} \alpha_{j k} \bar{\alpha}_{j k} b_{3 / 2}^{(2)}\left(\alpha_{j k}\right) \quad(k \neq j),
\end{aligned}
$$

$$
\begin{aligned}
& B_{j j}^{P}=-\frac{1}{4} n_{j} \sum_{k=1, k \neq j}^{N} \frac{m_{k}}{m_{c}+m_{j}} \alpha_{j k} \bar{\alpha}_{j k} b_{3 / 2}^{(1)}\left(\alpha_{j k}\right), \\
& B_{j k}^{P}=\frac{1}{4} n_{j} \frac{m_{k}}{m_{c}+m_{j}} \alpha_{j k} \bar{\alpha}_{j k} b_{3 / 2}^{(1)}\left(\alpha_{j k}\right) \quad(k \neq j),
\end{aligned}
$$

$$
\begin{aligned}
& A^{P}=\frac{1}{4} n \sum_{j=1}^{N} \frac{m_{j}}{m_{c}} \alpha_{j} \bar{\alpha}_{j} b_{3 / 2}^{(1)}\left(\alpha_{j}\right), \\
& A_{j}^{P}=-\frac{1}{4} n \frac{m_{j}}{m_{c}} \alpha_{j} \bar{\alpha}_{j} b_{3 / 2}^{(2)}\left(\alpha_{j}\right),
\end{aligned}
$$

$$
\begin{aligned}
B^{P} & =-\frac{1}{4} n \sum_{j=1}^{N} \frac{m_{j}}{m_{c}} \alpha_{j} \bar{\alpha}_{j} b_{3 / 2}^{(1)}\left(\alpha_{j}\right), \\
B_{j}^{P} & =\frac{1}{4} n \frac{m_{j}}{m_{c}} \alpha_{j} \bar{\alpha}_{j} b_{3 / 2}^{(1)}\left(\alpha_{j}\right),
\end{aligned}
$$


692

$$
\begin{aligned}
& h^{P}=e_{\text {free }}^{P} \sin \left(A^{P} t+\beta^{P}\right)+h_{0}^{P}(t), \\
& k^{P}=e_{\text {free }}^{P} \cos \left(A^{P} t+\beta^{P}\right)+k_{0}^{P}(t), \\
& p^{P}=I_{\text {free }}^{P} \sin \left(B^{P} t+\gamma^{P}\right)+p_{0}^{P}(t), \\
& q^{P}=I_{\text {free }}^{P} \cos \left(B^{P} t+\gamma^{P}\right)+q_{0}^{P}(t),
\end{aligned}
$$

${ }_{693}$ where $e_{\text {free }}^{P}, I_{\text {free }}^{P}, \beta^{P}$ and $\gamma^{P}$ are constants determined by the initial condi-

694 tions. The first terms of the right members of the equations 15, 16, 17 and 18

${ }_{695}$ correspond to the free oscillations while the terms $h_{0}^{P}(t), k_{0}^{P}(t), p_{0}^{P}(t)$ and $q_{0}^{P}(t)$ 
correspond to the forced oscillations:

$$
\begin{aligned}
& h_{0}^{P}=-\sum_{i=1}^{N} \frac{\nu_{i}^{P}}{A^{P}-g_{i}^{P}} \sin \left(g_{i}^{P} t+\beta_{i}^{P}\right), \\
& k_{0}^{P}=-\sum_{i=1}^{N} \frac{\nu_{i}^{P}}{A^{P}-g_{i}^{P}} \cos \left(g_{i}^{P} t+\beta_{i}^{P}\right), \\
& p_{0}^{P}=-\sum_{i=1}^{N} \frac{\mu_{i}^{P}}{B^{P}-f_{i}^{P}} \sin \left(f_{i}^{P} t+\gamma_{i}^{P}\right), \\
& q_{0}^{P}=-\sum_{i=1}^{N} \frac{\mu_{i}^{P}}{B^{P}-f_{i}^{P}} \cos \left(f_{i}^{P} t+\gamma_{i}^{P}\right),
\end{aligned}
$$

where the frequencies $g_{i}^{P}$ and $f_{i}^{P}$ are the eigenvalues (which we will call eigenfrequencies in the following) of the matrices $\mathbf{A}^{\mathbf{P}}$ and $\mathbf{B}^{\mathbf{P}}$ respectively. We also have $\nu_{i}^{P}=\sum_{j=1}^{N} A_{j}^{P} e_{j i}^{P}$ and $\mu_{i}^{P}=\sum_{j=1}^{N} B_{j}^{P} I_{j i}^{P}$ where $e_{j i}^{P}, I_{j i}^{P}, \beta_{i}^{P}$ and $\gamma_{i}^{P}$ are constants depending on the eigenvectors associated to the eigenfrequencies and on the initial conditions of the giant planets. These equations bring out the existence of resonances when the secular frequency $A^{P}$ and $B^{P}$ of the LD object, which depends on its semi-major axis, are equal to one of the eigenfrequencies $g_{i}^{P}$ and $f_{i}^{P}$ respectively. The resonances involving the frequencies $A^{P}$ and $g_{i}^{P}$ are called apsidal secular resonances whereas those involving the frequencies $B^{P}$ and $f_{i}^{P}$ are called nodal secular resonances.

Now, if we consider the disturbing function $R_{j}^{M D}$ of the $j$ th giant planet due to the MD and the disturbing function $R^{M D}$ of the $\mathrm{LD}$ object due to the MD, we have:

$$
\begin{aligned}
R_{j}^{M D} & =n_{j} a_{j}^{2}\left(\frac{1}{2} A_{j j}^{M D} e_{j}^{2}+\frac{1}{2} B_{j j}^{M D} I_{j}^{2}\right), \\
R^{M D} & =n a^{2}\left(\frac{1}{2} A^{M D} e^{2}+\frac{1}{2} B^{M D} I^{2}\right),
\end{aligned}
$$

where the terms with the superscript $M D$ denotes that they are due to the MD. As described in section 2, we can determine $R_{j}^{M D}$ and $R^{M D}$ computationnally. For example, figure 6 shows the apsidal part (top panel) and the nodal part (bottom panel) of the disturbing function of Jupiter due to the MD as a function of the eccentricity and the inclination of the planet respectively. 

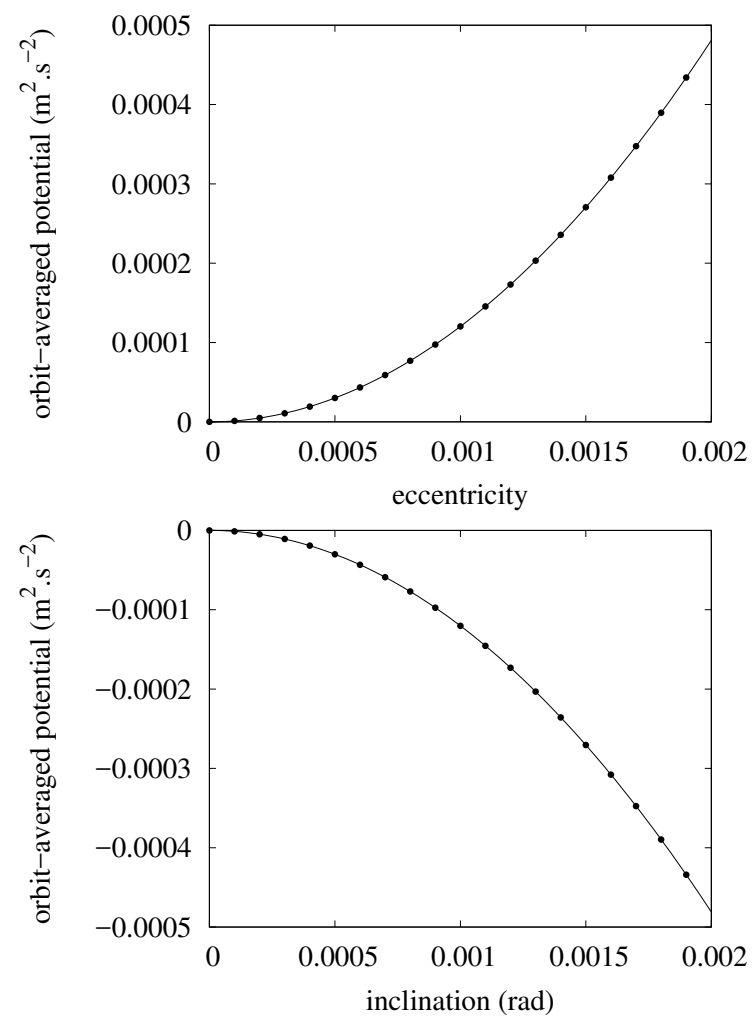

Figure 6: The disturbing function (or equivalently, the gravitational potential averaged on one orbital period along a fixed ellipse) of Jupiter (subscript $j=1$ ) due to the MD as a function of the eccentricity (top panel) and of the inclination (bottom panel). The dots are the values for which we have computed the averaged gravitational potential. The curves are the best fit functions of the form $R_{1} M D=\alpha e^{2}$ (top panel) and $R_{1} M D=\beta I^{2}$ (bottom panel), where $\alpha$ and $\beta$ are coefficients.

\section{Appendix B}

Here we describe the terms that we need to add to the user-defined acceleration and to the user-defined velocity in order to allow the longitudes of the node of the bodies to evolve as defined by the user in addition of the mutual interactions between the bodies. The components of the position vector $(x, y, z)$ and the components of the velocity vector $(\dot{x}, \dot{y}, \dot{z})$ can be expressed as a function of the osculating elements: 


$$
\begin{aligned}
x= & r \cos \Omega \cos (\omega+f)-r \cos i \sin \Omega \sin (\omega+f) \\
y= & r \sin \Omega \cos (\omega+f)+r \cos i \cos \Omega \sin (\omega+f) \\
z= & r \sin i \sin (\omega+f) \\
\dot{x}= & \cos \Omega(\dot{r} \cos (\omega+f)-r \dot{f} \sin (\omega+f)) \\
& -\sin \Omega(\dot{r} \cos i \sin (\omega+f)+r \dot{f} \cos i \cos (\omega+f)) \\
\dot{y}= & \sin \Omega(\dot{r} \cos (\omega+f)-r \dot{f} \sin (\omega+f)) \\
& +\cos \Omega(\dot{r} \cos i \sin (\omega+f)+r \dot{f} \cos i \cos (\omega+f)) \\
\dot{z}= & \dot{r} \sin i \sin (\omega+f)+r \dot{f} \sin i \cos (\omega+f),
\end{aligned}
$$

where $r$ is the heliocentric distance, $f$ the true anomaly, $i$ the inclination, $\omega$ the argument of the perihelion and $\Omega$ the longitude of the ascending node. Using the notations and the method of Lee and Peale (2002), we can obtain the terms needed to update the acceleration and the veolocity:

$$
\begin{aligned}
\left.\frac{d x}{d t}\right|_{\dot{\Omega}} & =\frac{\partial x}{\partial \Omega} \dot{\Omega} \\
& =(-r \sin \Omega \cos (\omega+f)-r \cos i \cos \Omega \sin (\omega+f)) \dot{\Omega} \\
& =-y \dot{\Omega} \\
\left.\frac{d y}{d t}\right|_{\dot{\Omega}} & =\frac{\partial y}{\partial \Omega} \dot{\Omega} \\
& =(r \cos \Omega \cos (\omega+f)-r \cos i \sin \Omega \sin (\omega+f)) \dot{\Omega} \\
& =\left.x \dot{\Omega} \quad \frac{d z}{d t}\right|_{\dot{\Omega}}=\frac{\partial z}{\partial \Omega} \dot{\Omega} \\
& =0 \\
\left.\frac{d \dot{x}}{d t}\right|_{\dot{\Omega}} & \frac{\partial \dot{x}}{\partial \Omega} \dot{\Omega} \\
= & -\sin \Omega(\dot{r} \cos (\omega+f)-r \dot{f} \sin (\omega+f)) \\
& -\cos \Omega(\dot{r} \cos i \sin (\omega+f)+r \dot{f} \cos i \cos (\omega+f))] \dot{\Omega} \\
= & -\dot{y} \dot{\Omega}
\end{aligned}
$$




$$
\begin{aligned}
\left.\frac{d \dot{y}}{d t}\right|_{\dot{\Omega}} & =\frac{\partial \dot{y}}{\partial \Omega} \dot{\Omega} \\
= & {[\cos \Omega(\dot{r} \cos (\omega+f)-r \dot{f} \sin (\omega+f))} \\
& -\sin \Omega(\dot{r} \cos i \sin (\omega+f)+r \dot{f} \cos i \cos (\omega+f))] \dot{\Omega} \\
= & \dot{x} \dot{\Omega}
\end{aligned}
$$

